\title{
A Note on the Guignard Constraint Qualification and the Guignard Regularity Condition in Vector Optimization
}

\author{
Giorgio Giorgi \\ Faculty of Economics, University of Pavia, Via S. Felice, Pavia, Italy \\ Email: ggiorgi@eco.unipv.it
}

Received December 12, 2012; revised February 14, 2013; accepted February 21, 2013

Copyright (C) 2013 Giorgio Giorgi. This is an open access article distributed under the Creative Commons Attribution License, which permits unrestricted use, distribution, and reproduction in any medium, provided the original work is properly cited.

\begin{abstract}
Some remarks are made on the use of the Abadie constraint qualification, the Guignard constraint qualifications and the Guignard regularity condition in obtaining weak and strong Kuhn-Tucker type optimality conditions in differentiable vector optimization problems.
\end{abstract}

Keywords: Constraint Qualifications; Regularity Conditions; Optimality Conditions; Vector Optimization Problems

\section{Introduction}

In discussing a gap between multiobjective optimization and scalar optimization (a gap first pointed out by Wang and Yang [1]), Aghezzaf and Hachimi [2] state that "in multiobjective optimization problems, many authors have derived the first-order and second-order necessary conditions under the Abadie constraint qualification, but never under the Guignard constraint qualification”. This deserves some comments. Indeed, some authors have proposed a suitable Guignard-Gould-Tolle constraint qualification (it would be better to speak of "GuignardGould-Tolle regularity condition", as it involves also the objective function, besides the constraint functions) in order to obtain a Karush-Kuhn-Tucker type multiplier rule for a Pareto optimization problem.

For example, Maeda [3] considers the following Pareto optimization problem

$$
\text { Min } f(x) \text {, subject to } g(x) \leq 0 \text {, }
$$

where $f: \mathbb{R}^{n} \rightarrow \mathbb{R}^{p}, g: \mathbb{R}^{n} \rightarrow \mathbb{R}^{m}$ are differentiable functions, and introduces the following "generalized Guignard constraint qualification” for this problem

$$
C\left(Q, x^{0}\right) \subset \bigcap_{i=1}^{p} \mathrm{cl}\left(\operatorname{conv}\left(T\left(Q^{i}, x^{0}\right)\right)\right),
$$

where $x^{0}$ is a feasible vector,

$$
\begin{gathered}
Q=\left\{x \in \mathbb{R}^{n}: g(x) \leq 0, f(x) \leq f\left(x^{0}\right)\right\} ; \\
Q^{i}=\left\{x \in \mathbb{R}^{n}: g(x) \leq 0, f_{k}(x) \leq f_{k}\left(x^{0}\right), k=1,2, \cdots, p \text { and } k \neq i\right\} ; \\
C\left(Q, x^{0}\right)=\left\{h \in \mathbb{R}^{n}: \nabla f_{i}\left(x^{0}\right) h \leq 0, i=1, \cdots, p ; \nabla g_{j}\left(x^{0}\right) h \leq 0, j \in I\left(x^{0}\right)\right\} ;
\end{gathered}
$$

$T\left(Q^{i}, x^{0}\right)$ is the Bouligand tangent cone or contingent cone to $Q^{i}$ at $x^{0}$ (see Definition 2) and $I\left(x^{0}\right)=\left\{j: g_{j}\left(x^{0}\right)=0\right\}$. Indeed, in the above constraint qualification (better: regularity condition) the inclusion means in fact equality.

Jimenez and Novo [4], Giorgi, Jimenez and Novo [5], [6], Giorgi and Zuccotti [7] have given similar, but more general results. What is true is that the GuignardGould-Tolle theory cannot be transferred "sic et simpliciter" from the scalar to the vector case. Indeed, it is well- known that if we have a scalar optimization problem

$$
\text { Min } f(x), x \in M \subset \mathbb{R}^{n},
$$

with $f: \mathbb{R}^{n} \rightarrow \mathbb{R}, f$ differentiable, and $x^{0}$ is a local solution of the said problem, then we have

$$
-\nabla f\left(x^{0}\right) \in\left(T\left(M, x^{0}\right)\right)^{*}
$$

( $A^{*}$ is the negative polar cone of the cone $A$ ). The result obtained by Guignard [8] seems at first sight more gen- 
eral, as Guignard claims that

$$
-\nabla f\left(x^{0}\right) \in\left(P\left(M, x^{0}\right)\right)^{*}
$$

where $P\left(M, x^{0}\right)=\operatorname{cl}\left(\operatorname{conv}\left(T\left(M, x^{0}\right)\right)\right)$ is the so-called pseudotangent cone to $M$ at $x^{0}$. However, this greater generality is only apparent, as it is true that for any cone $C$, it holds

$$
C^{*}=(\operatorname{cl}(\operatorname{conv}(C)))^{*},
$$

so we obtain

$$
\left(T\left(M, x^{0}\right)\right)^{*}=\left(P\left(M, x^{0}\right)\right)^{*} .
$$

Relation (1) obviously is equivalent to the inconsistency of the inequality

$$
\nabla f\left(x^{0}\right) y<0
$$

for $y \in T\left(M, x^{0}\right)$, or, equivalently, for $y \in P\left(M, x^{0}\right)$.

Now, consider a vector optimization problem (vop)

$$
\operatorname{Min} f(x), x \in M \subset \mathbb{R}^{n},
$$

where $f: \mathbb{R}^{n} \rightarrow \mathbb{R}^{p}$ is differentiable. When, as in the present paper, the ordering cone is $\mathbb{R}_{+}^{p}$, (vop) is also known as Pareto optimization problem. We recall some basic notions and definitions.

\section{Definition 1.}

A vector $x^{0} \in M$ is said to be a weak Pareto optimal point for (vop) if there does not exist another vector $x \in M$ such that $f_{k}(x)<f_{k}\left(x^{0}\right)$ for all $k=1, \cdots, p$. A vector $x^{0}$ is said to be a Pareto optimal point for (vop) if there does not exist another vector $x \in M$ such that $f_{k}(x) \leq f_{k}\left(x^{0}\right)$ for all $k=1, \cdots, p$, with $f_{k}(x)<$ $f_{k}\left(x^{0}\right)^{k}$ for at least one index. A vector $x^{0} \in M$ is a local weak Pareto optimal point (respectively, a local Pareto optimal point) if the above definitions hold with respect to $M \cap B\left(x^{0}, \delta\right)$, where $B\left(x^{0}, \delta\right)$ is a suitable neighborhood of $x^{0}$.

\section{Definition 2.}

Let $M \subset \mathbb{R}^{n}$. The contingent cone at $x^{0} \in \mathrm{cl}(M)$ or Bouligand tangent cone at $x^{0}$ is:

$$
\begin{aligned}
T\left(M, x^{0}\right)= & \left\{y \in \mathbb{R}^{n}: \exists\left\{y^{n}\right\} \rightarrow y, \exists\left\{t_{n}\right\}, t_{n} \rightarrow 0^{+},\right. \\
& \text {such that } \left.x^{0}+t_{n} y^{n} \in M\right\}
\end{aligned}
$$

or, equivalently,

$$
\begin{aligned}
T\left(M, x^{0}\right)= & \left\{y \in \mathbb{R}^{n}: \exists\left\{\lambda_{n}\right\} \subset \mathbb{R}_{+}, \exists\left\{x^{n}\right\} \subset M, x^{n} \rightarrow x^{0},\right. \\
& \text { such that } \left.\lambda_{n}\left(x^{n}-x^{0}\right) \rightarrow y\right\} .
\end{aligned}
$$

It is well-known that this cone is closed, but not necessarily convex (see, e.g., Aubin and Frankowska [9], Bazaraa and Shetty [10]).
It can be proved that if $x^{0}$ is a local weak optimal point for (vop), then we have the relation (see, e.g., Bigi ([11], Corley [12], Giorgi and Zuccotti [13], Staib [14])

$$
\nabla f\left(x^{0}\right) v \notin-\operatorname{int}\left(\mathbb{R}_{+}^{p}\right), \forall v \in T\left(M, x^{0}\right),
$$

i.e. the system

$$
\nabla f_{i}\left(x^{0}\right) v<0, i=1, \cdots, p,
$$

has no solution for $v \in T\left(M, x^{0}\right)$, i.e. it holds

$$
\operatorname{Max}_{k=1, \cdots, p}\left\{\nabla f_{k}\left(x^{0}\right) v\right\} \geq 0, \forall v \in T\left(M, x^{0}\right) .
$$

One may wonder if the system (3) (for $p>1$ ) is also inconsistent for $v \in \mathrm{cl}\left(\operatorname{conv}\left(T\left(M, x^{0}\right)\right)\right)$, as it holds for $p=1$. The answer is: no, as shown by Wang and Yang [1] with a numerical example (a misprint in the example has been corrected by Castellani and Pappalardo [15]). This is the "gap", with reference to a result of Guignard to which the title of the paper of Wang and Yang in its turn makes reference.

This note is organized as follows.

In Section 2 we give short proofs of the weak KuhnTucker type necessary optimality conditions, for a Pareto optimization problem with both inequality and equality constraints, under the Abadie constraint qualification, and of the strong Kuhn-Tucker type conditions, for the same problem, under a Guignard regularity condition.

In Section 3 we make some further comments on the said "gap" between scalar optimization problems and vector optimization problems.

The conclusions are briefly expounded in Section 4.

\section{First Order Necessary Conditions: Abadie Constraint Qualification, Guignard Regularity Condition}

The feasible set of (vop) is, from now on, specified by inequality and equality constraints. More precisely, we consider the problem

$$
\text { (vop) } 1 \quad \text { Min } f(x), x \in M \text {, }
$$

where

$$
\begin{aligned}
M= & \left\{x \in \mathbb{R}^{n}: g_{i}(x) \leq 0, i=1, \cdots, m ;\right. \\
& \left.h_{j}(x)=0, j=1, \cdots, r\right\},
\end{aligned}
$$

$f: \mathbb{R}^{n} \rightarrow \mathbb{R}^{p}, g: \mathbb{R}^{n} \rightarrow \mathbb{R}^{m}$ are differentiable at least in a neighborhood of the feasible point $x^{0}$, and $h: \mathbb{R}^{n} \rightarrow \mathbb{R}^{r}$ is continuously differentiable at least in the same neighborhood of $x^{0}$. We denote by $I\left(x^{0}\right)$ the index set of the active constraints $g_{i}, i=1, \cdots, m$, at $x^{0} \in M$, i.e.

$$
I\left(x^{0}\right)=\left\{i: g_{i}\left(x^{0}\right)=0\right\} \text {. }
$$

Let $x^{0} \in M$; the cone 


$$
\begin{aligned}
K\left(x^{0}\right)= & \left\{y \in \mathbb{R}^{n}: \nabla g_{i}\left(x^{0}\right) y \leq 0, \forall i \in I\left(x^{0}\right) ;\right. \\
& \left.\nabla h_{j}\left(x^{0}\right) y=0, \forall j=1, \cdots, r\right\}
\end{aligned}
$$

is called the linearizing cone at $x^{0}$ for (vop) $)_{1}$.

The cone

$$
\begin{aligned}
K^{\circ}\left(x^{0}\right)= & \left\{y \in \mathbb{R}^{n}: \nabla g_{i}\left(x^{0}\right) y \leq 0, \forall i \in I\left(x^{0}\right) ;\right. \\
& \left.\nabla h_{j}\left(x^{0}\right) y=0, \forall j=1, \cdots, r\right\}
\end{aligned}
$$

is called the weak linearizing cone at $x^{0}$ or cone of decreasing directions at $x^{0}$ for (vop) $)_{1}$.

Lemma 1. (see Giorgi [16])

Let $x^{0} \in M$ and let $f, g$ and $h$ verify the previous differentiability assumptions. Then we have:

1) $T\left(M, x^{0}\right) \subset K\left(x^{0}\right)$;

2) if the Jacobian matrix $\nabla h\left(x^{0}\right)$ has full rank, it holds $K^{\circ}\left(x^{0}\right) \subset T\left(M, x^{0}\right)$;

3) if, moreover, $K^{\circ}\left(x^{0}\right) \neq \varnothing$, then it holds

$$
\operatorname{cl}\left(K^{\circ}\left(x^{0}\right)\right)=T\left(M, x^{0}\right)=K\left(x^{0}\right) .
$$

We are now ready to prove in a short way a Fritz John-type optimality condition for (vop) ${ }_{1}$.

\section{Lemma 2.}

Suppose that the Jacobian matrix $\nabla h\left(x^{0}\right)$ has full rank. Let $x^{0} \in M$ be a local weak Pareto optimal point for (vop) ${ }_{1}$. Then the system

$$
\left\{\begin{array}{l}
\nabla f_{k}\left(x^{0}\right), v<0, k=1, \cdots, p, \\
\nabla g_{i}\left(x^{0}\right), v<0, i \in I\left(x^{0}\right), \\
\nabla h_{j}\left(x^{0}\right), v=0, j=1, \cdots, r,
\end{array}\right.
$$

has no solution $v \in \mathbb{R}^{n}$.

Proof.

Apply Lemma 1 to the optimality condition (3).

\section{Theorem 1.}

If $x^{0} \in M$ is a local weak Pareto optimal point for (vop) $)_{1}$, then there exist vectors $\theta \in \mathbb{R}^{p}, \lambda \in \mathbb{R}^{m}$, and $\mu \in \mathbb{R}^{r}$, not all zero, such that

$$
\begin{gathered}
\sum_{k=1}^{p} \theta_{k} \nabla f_{k}\left(x^{0}\right)+\sum_{i=1}^{m} \lambda_{i} \nabla g_{i}\left(x^{0}\right)+\sum_{j=1}^{r} \mu_{j} \nabla h_{j}\left(x^{0}\right)=0, \\
\lambda_{i} g_{i}\left(x^{0}\right)=0, i=1, \cdots, m, \\
\theta_{k} \geq 0, k=1, \cdots, p ; \lambda_{i} \geq 0, i=1, \cdots, m .
\end{gathered}
$$

Proof.

If the gradients $\nabla h_{j}\left(x^{0}\right), j=1, \cdots, r$, are linearly dependent, just set $\theta=0, \lambda=0$ and choose a nonzero vector $\mu \in \mathbb{R}^{r}$ such that

$$
\sum_{j=1}^{r} \mu_{j} \nabla h_{j}\left(x^{0}\right)=0 .
$$

If the above gradients are linearly independent, the thesis follows from Lemma 2, applying the Motzkin alternative theorem (see, e. g., Mangasarian [17]) and setting $\lambda_{i}=0$ for all $i \notin I\left(x^{0}\right)$.

A Karush-Kuhn-Tucker-type optimality conditions for (vop)1, by means of the Abadie constraint qualification (acq), has been obtained by Lin [18] and by Singh [19]; however, their proofs work only if $x^{0}$ is a weak Pareto optimal point for (vop) $)_{1}$, and not a local weak Pareto optimal point. The flaw is corrected in Marusciac [20]; see also the errata corrige of Singh [19], who, however, does not justify his rectification; see also the paper of Wang [21], more specific on this point. We give here a simple proof of the result of Wang.

\section{Definition 3.}

The constraint set $M$ satisfies the Abadie constraint qualification (acq) at $x^{0} \in M$ if

$$
K\left(x^{0}\right)=T\left(M, x^{0}\right) .
$$

Due to relation 1) of Lemma 1, the (acq) can be written also as an inclusion

$$
K\left(x^{0}\right) \subset T\left(M, x^{0}\right) .
$$

Singh [19] claims that his version, as an inclusion, of the (acq) is more general than the one proposed by Marusciac [20] as an equality.

\section{Lemma 3.}

Let $x^{0} \in M$ be a local weak Pareto optimal point for (vop) $)_{1}$, and suppose that the (acq) holds at $x^{0}$. Then the system

$$
\left\{\begin{array}{l}
\nabla f_{k}\left(x^{0}\right) v<0, k=1, \cdots, p, \\
\nabla g_{i}\left(x^{0}\right) v \leq 0, i \in I\left(x^{0}\right), \\
\nabla h_{j}\left(x^{0}\right) v=0, j=1, \cdots, r,
\end{array}\right.
$$

has no solution $v \in \mathbb{R}^{n}$.

Proof.

Suppose, on the contrary, that the above system has a solution $v \in \mathbb{R}^{n}$. Then, the (acq) implies that $v \in T\left(M, x^{0}\right)$; thanks to relation (3) it will hold $\nabla f_{k}\left(x^{0}\right) v \geq 0$ for at least one index $k$, contradicting the relations of the system.

Applying to system (8) the Motzkin theorem of the alternative, we get the following (weak) Karush-KuhnTucker-type multiplier rule for (vop) ${ }_{1}$.

Theorem 2.

Let $x^{0} \in M$ be a local weak Pareto optimal point for ( vop) $)_{1}$ and let the (acq) hold at $x^{0}$. Then there exist vectors $\theta \in \mathbb{R}_{+}^{p}, \lambda \in \mathbb{R}_{+}^{m}$, and $\mu \in \mathbb{R}$, with $\theta \neq 0$, such that (5), (6) and (7) hold.

As the cone $K\left(x^{0}\right)$ is polyhedric, the (acq) implies that $T\left(M, x^{0}\right)$ is a convex cone. If we substitute $T\left(M, x^{0}\right)$ with the closure of its convex hull, we obtain 
the Guignard-Gould-Tolle constraint qualification (Guignard [8] Gould and Tolle [22])

$$
K\left(x^{0}\right)=\operatorname{cl}\left(\operatorname{conv}\left(T\left(M, x^{0}\right)\right)\right)=P\left(M, x^{0}\right),
$$

but, as already remarked in the Introduction, this more general constraint qualification does not enable to obtain, for $p>1$, the multiplier rule of Theorem 2, unless to make further assumptions on $T\left(M, x^{0}\right)$ or on the objective function. See the next Section 3.

If we want to obtain a strong Karush-Kuhn-Tucker type optimality condition for (vop) $)_{1}$, i.e. a multiplier rule (5), (6) and (7), where $\theta>0$ in (5), we have to impose some condition, where also the objective function is considered. We prefer, in this case, to speak of regularity conditions, instead of constraint qualifications. The condition considered by Maeda [3] and reported in the Introduction of the present paper, is therefore a regularity condition. For other regularity conditions for (vop) ${ }_{1}$ and their relationships, see also Jimenez and Novo [4] and Giorgi and Zuccotti [7].

We now consider a slightly modified version of the Guignard regularity condition proposed by the above authors. See also Bigi [11].

\section{Definition 4.}

Let $x^{0} \in M$; then the set

$$
F\left(x^{0}\right)=\left\{v \in \mathbb{R}^{n}: \nabla_{k}\left(x^{0}\right) v \leq 0, k=1, \cdots, p\right\}
$$

is the cone of descent directions for $f$ at $x^{0}$. Given any $s \in P=\{1, \cdots, p\}$, the set

$$
M_{s}=\left\{x \in M: f_{k}(x)-f_{k}\left(x^{0}\right)<0, k \in P, k \neq s\right\} \cup\left\{x^{0}\right\}
$$

is the set of all feasible points, which do not allow $x^{0}$ to be a weak minimum point for (vop) ${ }_{1}$ when the component $f_{s}$ is dropped from $f$.

\section{Definition 5.}

Let $x^{0} \in M$ Then the (modified) Guignard-GouldTolle regularity condition (ggtrc) holds at $x^{0}$ if

$$
F\left(x^{0}\right) \cap K\left(x^{0}\right)=\bigcap_{s=1}^{p} \mathrm{cl}\left(\operatorname{conv}\left(T\left(M_{s}, x^{0}\right)\right)\right) .
$$

\section{Lemma 4.}

Suppose that $x^{0} \in M$ is a local weak Pareto optimal point for (vop) $)_{1}$ and that (ggtrc) holds at $x^{0}$. Then for each $k \in P=\{1, \cdots, p\}$ the following system

$$
\left\{\begin{array}{l}
\nabla f_{s}\left(x^{0}\right), v<0, \\
\nabla f_{k}\left(x^{0}\right), v \leq 0, k \in P, k \neq s, \\
\nabla g_{i}\left(x^{0}\right), v \leq 0, i \in I\left(x^{0}\right), \\
\nabla h_{j}\left(x^{0}\right), v=0, j=1, \cdots, r
\end{array}\right.
$$

has no solution $v \in \mathbb{R}^{n}$.
Proof.

On the contrary suppose that there exists $\bar{S} \in P$ such that (10) has a solution. Therefore, (ggtrc) implies that $v \in \operatorname{cl}\left(\operatorname{conv}\left(T\left(M, x^{0}\right)\right)\right)$. Since the definition of local weak Pareto optimal point for (vop) $)_{1}$ implies that $x^{0}$ is a local minimum point of the scalar function $f_{s}$ over $M_{s}$, then we get the inequality $\nabla f_{s}\left(x^{0}\right) v \geq 0$, in contradiction with the assumption.

The previous lemma, which states the impossibility of $p$ systems, enables us to obtain a strong Karush-KuhnTucker-type multiplier rule for (vop) $)_{1}$.

\section{Theorem 3.}

Suppose that $x^{0} \in M$ is a local weak Pareto optimal point for (vop) $)_{1}$ and that (ggtrc) holds at $x^{0}$. Then there exists vectors $\theta \in \mathbb{R}_{+}^{p}, \lambda \in \mathbb{R}_{+}^{m}$ and $\mu \in \mathbb{R}^{r}$, with $\theta_{k}>0$, for each $k \in P=\{1, \cdots, p\}$, such that (5), (6) and (7) hold.

Proof.

The proof is immediate, by applying the Motzkin theorem of the alternative to system (10), for each $s \in P$ and summing up the resulting multipliers.

We note that Maeda [3] has proposed a slightly weaker condition than (ggtrc), generalized to (vop) 1 by Jimenez and Novo [4], by Giorgi, Jimenez and Novo [5,6], and by Giorgi and Zuccotti [7], but this weaker regularity condition "works" for local Pareto optimal points and not for the weak ones. Finally, we remark that (generalizing some approaches of Bigi and Pappalardo [23]) Maciel, Santos and Sottosanto [24] and Giorgi and Zuccotti [7] have studied the following question: which regularity condition for (vop) $)_{1}$ is both necessary and sufficient to obtain that $\theta_{k}>0, k \in P$, for all triplets of multipliers $(\theta, \lambda, \mu)$ which satisfy relations (5), (6) and (7)? Let us denote by $F J\left(x^{0}\right)$ the above set of multipliers, i.e. the set of all Fritz John multipliers for (vop) ${ }_{1}$, and let us introduce the following generalization of the Mangasarian-Fromovitz constraint qualification.

\section{Definition 6.}

Let $x^{0} \in M$, then the Mangasarian-Fromovitz regularity condition (mfrc) for (vop) $)_{1}$ is satisfied at $x^{0}$ if:

1) the vectors $\nabla h_{j}\left(x^{0}\right), j=1, \cdots, r$, are linearly independent;

2) for all $s \in P=\{1, \cdots, p\}$ the system

$$
\left\{\begin{array}{l}
\nabla f_{k}\left(x^{0}\right), v<0, k \in P, k \neq s, \\
\nabla g_{i}\left(x^{0}\right), v<0, i \in I\left(x^{0}\right), \\
\nabla h_{j}\left(x^{0}\right), v=0, j=1, \cdots, r
\end{array}\right.
$$

has solution $v \in \mathbb{R}^{n}$.

It can be proved the following result (see Maciel, Santos and Sottosanto [24], Giorgi and Zuccotti [7]).

Theorem 4.

Let $x^{0} \in M$ and let $F J\left(x^{0}\right) \neq \varnothing$. Then in (vop) $)_{1}$ we have $(\theta, \lambda, \mu) \in F J\left(x^{0}\right)$ with $\theta_{k}>0$ for all $k \in P$, if 
and only if (mfrc) holds at $x^{0}$.

In this section we have given a simple and short proof of the Fritz John type and of the weak Kuhn-Tucker type necessary optimality conditions for the problem (vop) under the Abadie constraint qualification. This completes and improves what previously proved by Singh [19]. We give also a short proof of the strong Kuhn-Tucker type conditions for (vop) ${ }_{1}$, under a Guignard regularity condition. This improves what previously proved by Maeda [3].

\section{Again on the "Gap" between Scalar Problems and Vector Problems}

We have described in the Introduction the "gap" occurring between scalar and vector optimization problems, generated by the use of the classical Guignard-GouldTolle constraint qualification. We have also mentioned this "gap" in the previous section. An obvious sufficient condition to remove this "gap" is that the cone $T\left(M, x^{0}\right)$ is convex; this condition has been envisaged by Wang and Yang [1], who, however, did not go further in the discussion. It is well-known that $T\left(M, x^{0}\right)$ is convex when $M$ is a convex set. As the structure of the contingent cone $T\left(M, x^{0}\right)$, as of all the other first-order local cone approximations, depends only from the structure of $M$ arouund $x^{0}$ (see, e.g., Elster and Thierfelder [25]), we can state that $T\left(M, x^{0}\right)$ is convex also when $M$ is locally convex at $x^{0}$, i.e. there exists a neighborhood of $x^{0}, N\left(x^{0}\right)$, such that $M \cap N\left(x^{0}\right)$ is a convex set. This is no longer true when $M$ is only star-shaped at $x^{0}$, i.e. $\lambda x^{0}+(1-\lambda) x \in M, \forall x \in M$, contrary to what stated in Giorgi and Guerraggio [26] and to what seems stated in Palata [27] and in Staib [14].

If $M$ is star-shaped at $x^{0} \in M$ it holds that $T\left(M, x^{0}\right)=A\left(M, x^{0}\right)$, where

$$
\begin{aligned}
A\left(M, x^{0}\right)= & \left\{v \in \mathbb{R}^{n}: \exists \varphi: \mathbb{R}_{+} \rightarrow \mathbb{R}^{n},\right. \\
& \exists \delta>0 \text { such that } \varphi(\theta) \in M, \\
& \left.\forall \theta \in(0, \delta), \varphi(\theta)=x^{0}, \varphi^{\prime}(0)=v\right\}
\end{aligned}
$$

is the cone of the attainable directions or Kuhn-Tucker cone at $x^{0} \in \mathrm{cl}(M)$. So, when $M$ is star-shaped at $x^{0} \in M$, the (acq) and the Kuhn-Tucker constraint qualification (ktcq)

$$
K\left(x^{0}\right)=A\left(M, x^{0}\right)
$$

coincide (see Bazaraa and Shetty [10], who, however, do not recognize that the cone $A\left(M, x^{0}\right)$ is closed).

It is also well-known that if $T\left(M, x^{0}\right)$ equals the Clarke tangent cone

$$
\begin{array}{r}
T C\left(M, x^{0}\right)=\left\{v \in \mathbb{R}^{n}: \forall t_{n} \rightarrow 0^{+}, \forall x^{n} \rightarrow x^{0}, x^{n} \in M,\right. \\
\left.\exists v^{n} \rightarrow v \text { such that } x^{n}+t_{n} v^{n} \in M, \forall n\right\},
\end{array}
$$

then $T\left(M, x^{0}\right)$ is convex, being $T C\left(M, x^{0}\right)$ always closed and convex. In this case Penot [28] qualifies the set $M$ as tangentially regular at $x^{0}$. We follow the treatment of Aubin and Frankowska [9].

\section{Definition 7.}

We say that a closed subset $M \subset \mathbb{R}^{n}$ is sleek at $x^{0} \in M$ if the multivalued map $x \rightrightarrows T\left(M, x^{0}\right), x \in M$, is lower semicontinuous at $x^{0}$.

Theorem 5. (Aubin and Frankowska [9])

Let $M$ be a closed subset of $\mathbb{R}^{n}$ and $x^{0} \in M$. If $M$ is sleek at $x^{0}$, then the contingent cone $T\left(M, x^{0}\right)$ and the Clarke tangent cone $T C\left(M, x^{0}\right)$ coincide and consequently $T\left(M, x^{0}\right)$ is convex.

Another possibility to remove the "gap" is suggested by Castellani and Pappalardo [15], who impose that the objective function $f$ is convexlike ad refer a result of $\mathrm{Li}$ and Wang [29]. This can be proved directly in the following way.

\section{Definition 8.}

A function $f: \mathbb{R}^{n} \rightarrow \mathbb{R}^{p}$ is convexlike on the nonempty set $X \subset \mathbb{R}^{n}$ if for any $x^{1}, x^{2} \in X$ and any $\lambda \in[0,1)$ there exists $x^{3} \in X$ such that

$$
\lambda f\left(x^{1}\right)+(1-\lambda) f\left(x^{2}\right)-f\left(x^{3}\right) \in \mathbb{R}_{+}^{p} .
$$

Note that in the above definition $x^{3}$, which usually depends from $x^{1}, x^{2}$ and $\lambda$, is not required to be the convex combination of $x^{1}$ and $x^{2}$. Note, moreover, that if $p=1$, then any real-valued function is convexlike. Obviously, all convex functions $f: \mathbb{R}^{n} \rightarrow \mathbb{R}^{p}$ are convexlike; this is only a sufficient condition. For other sufficient conditions see Elster and Nehse [30]. See also Hayashi and Komiya [31] and Jeyakumar [32] for applications of convexlike functions to optimization problems.

A straightforward consequence of Definition 8 is the following characterization.

\section{Theorem 6.}

The function $f: \mathbb{R}^{n} \rightarrow \mathbb{R}^{p}$ is convexlike on $X \subset \mathbb{R}^{n}$ if and only if the set $f(X)+\mathbb{R}_{+}^{p}$ is convex.

\section{Theorem 7.}

Suppose that $f$ is convexlike on $M \subset \mathbb{R}^{n}$. If $x^{0} \in M$ is a weak Pareto optimal point for (vop), then there exists a nonnegative nonzero vector $\theta \in \mathbb{R}^{p}$ such that $x^{0}$ is a minimum point of the scalar problem

$$
\operatorname{Min}\{\theta f(x), x \in M\} .
$$

Proof.

By assumption $f\left(x^{0}\right) \notin f(M)+\operatorname{int}\left(\mathbb{R}_{+}^{p}\right)$. The convexlikeness assumption on $f$ implies that $f(M)+\operatorname{int}\left(\mathbb{R}_{+}^{p}\right)$ is convex; therefore the usual separation theorem implies the existence of a nonzero vector $\theta \in \mathbb{R}^{p}$ and of a scalar $\alpha \in \mathbb{R}$ such that

$$
\theta f\left(x^{0}\right) \leq \alpha \leq \theta(f(x)+d)
$$


for all $d \in \operatorname{int}(M)$ and for all $x \in M$. Therefore, considering $x=x^{0}$, we obtain $\theta \geq 0$; given any $d \in \operatorname{int}\left(\mathbb{R}_{+}^{p}\right)$ and considering $t d$ as $t \rightarrow 0$, we get $\theta f(x) \geq \theta f\left(x^{0}\right)$ and therefore the thesis follows.

We note that in the proof of the above ressult we use the property that $f(M)+\operatorname{int}\left(\mathbb{R}_{+}^{p}\right)$ is a convex set; this weaker condition is equivalent that $f: \mathbb{R}^{n} \rightarrow \mathbb{R}^{p}$ is subconvexlike on $M$ (see $\mathrm{Li}$ and Wang [29]). The "scalarization theorem" just proved allows us to state the following result (recall that for $p=1$ the Guignard-GouldTolle theory is consistent).

\section{Theorem 8.}

Suppose that $f: \mathbb{R}^{n} \rightarrow \mathbb{R}^{p}$ is convexlike (or subconvexlike) on $M$. If $x^{0} \in M$ is a weak Pareto optimal point for (vop), then

$$
\begin{aligned}
& \underset{k \in P}{\operatorname{Max}}\left\{\nabla f_{k}\left(x^{0}\right) v\right\} \geq 0, \\
& \forall v \in \operatorname{cl}\left(\operatorname{conv}\left(T\left(M, x^{0}\right)\right)\right) .
\end{aligned}
$$

Similarly, with reference to (vop) ${ }_{1}$, we can assert the following result.

\section{Theorem 9.}

Suppose that $x^{0} \in M$ is a weak Pareto optimal point for (vop) $)_{1}$ and that $f: \mathbb{R}^{n} \rightarrow \mathbb{R}^{p}$ is convexlike (or subconvexlike) on $M$. Suppose that the Guignard-GouldTolle constraint qualification (9) holds at $x^{0}$. Then, there exist multipliers $\theta \in \mathbb{R}_{+}^{p}, \theta \neq 0, \lambda \in \mathbb{R}_{+}^{m}$ and $\mu \in \mathbb{R}^{r}$ such that (5), (6) and (7) hold.

Another condition which allows to apply to (vop) ${ }_{1}$ the (ggtcq) and which entails the objective function $f$ is given in the following theorem.

\section{Theorem 10.}

Let $x^{0} \in M$ be a weak Pareto optimal point for (vop) ${ }_{1}$; suppose that $x^{0}$ verifies the (ggtcq) and that there exists a nonnegative vector $\theta \in \mathbb{R}_{+}^{p}, \theta \neq 0$, such that

$$
-\theta \nabla f\left(x^{0}\right) \in\left(T\left(M, x^{0}\right)\right)^{*} \text {. }
$$

Then $x^{0}$ verifies the conditions (5), (6) and (7).

Proof.

The (ggtcq) can be equivalently written in the form $\left(T\left(M, x^{0}\right)\right)^{* *}=K\left(x^{0}\right)$, or $\left(T\left(M, x^{0}\right)\right)^{*}=\left(K\left(x^{0}\right)\right)^{*}$. On the other hand, being $K\left(x^{0}\right)$ a polyhedral cone, determined by the vectors $\nabla g_{i}\left(x^{0}\right), i \in I\left(x^{0}\right)$ and $\nabla h_{j}\left(x^{0}\right)$, $j=1, \cdots, r$, its polar will be given by

$$
\begin{gathered}
\left(K\left(x^{0}\right)\right)^{*}=\left\{\sum_{i \in I\left(x^{0}\right)} \lambda_{i} \nabla g_{i}\left(x^{0}\right)+\sum_{j=1}^{r} \mu_{j} \nabla h_{j}\left(x^{0}\right),\right. \\
\left.\lambda_{i} \geq 0, \forall i \in I\left(x^{0}\right)\right\} .
\end{gathered}
$$

Therefore, being $-\theta \nabla f\left(x^{0}\right) \in\left(T\left(M, x^{0}\right)\right)^{*}$, we can write

$$
\begin{gathered}
-\theta \nabla f\left(x^{0}\right)=\sum_{i \in I\left(x^{0}\right)} \lambda_{i} \nabla g_{i}\left(x^{0}\right)+\sum_{j=1}^{r} \mu_{j} \nabla h_{j}\left(x^{0}\right), \\
\lambda_{i} \geq 0, \forall i \in I\left(x^{0}\right),
\end{gathered}
$$

i.e. the conditions (5), (6) and (7), by choosing $\lambda_{i}=0$ for $i \notin I\left(x^{0}\right)$.

We remark that if $x^{0} \in M$ is a weak Pareto opyimal point for (vop) $)_{1}$, then, for each convex cone $S$, with $S \subset T\left(M, x^{0}\right)$, there exists a nonnegative vector $\theta \in \mathbb{R}_{+}^{p}$, $\theta \neq 0$, such that $-\theta \nabla f\left(x^{0}\right) \in S^{*}$, The proof is left to the reader (apply the classical theorem on separation of convex sets). Note that when $S=T\left(M, x^{0}\right)$ we obtain the result already observed at the beginning of this Section and obtain also the result stated in the previous theorem.

In this section we have investigated the reasons for the existence of a gap between a scalar programming problem and a multiobjective programming problem (vop), gap which has its origins in the use of the classical Guignard-Gould-Tolle constraint qualification. We have given some proposals to remove the said gap.

\section{Conclusions}

The aim of the present paper is twofold. On one side we present simple and brief optimality conditions of the Fritz John type and of the Kuhn-Tucker type (both weak and strong) for a Pareto multiobjective programming problem with both inequality and equality constraints. On the other side we investigate the existence of a gap, originated by the use of the classical Guignard constraint qualification, between a scalar nonlinear programming problem and the Pareto problem described above.

The author thanks an anonymous referee for his suggestions.

\section{REFERENCES}

[1] S. Y. Wang and F. M. Yang, "A Gap between Multiobjective Optimization and Scalar Optimization,” Journal of Optimization Theory and Applications, Vol. 68, No. 2, 1991, pp. 389-391. doi:10.1007/BF00941577

[2] B. Aghezzaf and M. Hachimi, "On a Gap between Multiobjective Optimization and Scalar Optimization,” Journal of Optimization Theory and Applications, Vol. 109, No. 2, 2001, pp. 431-435. doi:10.1023/A:1017574608034

[3] T. Maeda, “Constraint Qualifications in Multiobjective Optimization Problems: Differentiable Case," Journal of Optimization Theory and Applications, Vol. 80, No. 3, 1994, pp. 483-500. doi:10.1007/BF02207776

[4] B. Jimenez and V. Novo, "Cualificaciones de Restricciones en Problemas de Optimización Vectorial Diferenciables," XVI CEDYA Congreso de Ecuaciones Diferenciales y Aplicaciones, VI CMA Congreso de Matemática Aplicada, Vol. 1, 1999, pp. 727-734.

[5] G. Giorgi, B. Jimenez and V. Novo, "On Constraint 
Qualifications in Directionally Differentiable Multiobjective Optimization Problems," RAIRO-Operations Research, Vol. 38, No. 3, 2004, pp. 255-274.

doi:10.1051/ro:2004023

[6] G. Giorgi, B. Jimenez and V. Novo, "Strong KuhnTucker Conditions and Constraint Qualifications in Locally Lipschitz Multiobjective Optimization,” TOP, Vol. 17, No. 2, 2009, pp. 288-304. doi:10.1007/s11750-008-0058-z

[7] G. Giorgi and C. Zuccotti, "Again on Regularity Conditions in Differentiable Vector Optimization,” Annals of the University of Bucharest (Mathematical Series), Vol. LX, No.2, 2011, pp. 157-177.

[8] M. Guignard, "Generalized Kuhn-Tucker Conditions for Mathematical Programming Problems in a Banach Space," SIAM Journal on Control, Vol. 7, No. 2, 1969, pp. 232241. doi:10.1137/0307016

[9] J. P. Aubin and H. Frankowska, "Set-Valued Analysis," Birkhäuser, Boston, 1990

[10] M. S. Bazaraa and C. M. Shetty, "Foundations of Optimization,” Springer Verlag, Berlin, 1976. doi:10.1007/978-3-642-48294-6

[11] G. Bigi, “Optimality and Lagrangian Regularity in Vector Optimization,” Ph.D. Thesis, University of Pisa, Pisa, 1999.

[12] H. W. Corley, "On Optimality Conditions for Maximizations with Respect to Cones," Journal of Optimization Theory and Applications, Vol. 46, No. 1, 1985, pp. 67-78. doi:10.1007/BF00938760

[13] G. Giorgi and C. Zuccotti, "On the Use of Some Tangent Cones and Sets in Vector Optimization,” Report N. 169, Università di Pavia, Pavia, 2012.

[14] T. Staib, “On Necessary and Sufficient Optimality Conditions for Multicriteria Optimization Problems," ZORMethods and Models of Operations Research, Vol. 35, 1991, pp. 231-248.

[15] M. Castellani and M. Pappalardo, "About a Gap between Multiobjective Optimization and Scalar Optimization," Journal of Optimization Theory and Applications, Vol. 109, No. 2, 2001, pp. 437-439. doi:10.1023/A:1017526724872

[16] G. Giorgi, "Remarks on the Fritz John Conditions for Problems with Inequality and Equality Constraints," International Journal of Pure and Applied Mathematics, Vol. 71, 2011, pp. 643-657.

[17] O. L. Mangasarian, “Nonlinear Programming,” McGrawHill, New York, 1969.

[18] J. G. Lin, "Maximal Vectors and Multi-Objective Optimization," Journal of Optimization Theory and Applications, Vol. 18, No. 1, 1976, pp. 41-64. doi:10.1007/BF00933793

[19] C. Singh, "Optimality Conditions in Multiobjective Differentaible Programming," Journal of Optimization Theory and Applications, Vol. 53, No. 1, 1987, pp. 115-123.
C. Singh, "Errata Corrige," Journal of Optimization Theory and Applications, Vol. 57, No. 2, 1988, p. 369. doi:10.1007/BF00938547

[20] I. Marusciac, “On Fritz John Type Optimality Criterion in Multiobjective Optimization,” L'Analyse Numérique et la Théorie de l'Approximation, Vol. 11, 1982, pp. 109-114.

[21] S. Y. Wang, “A Note on Optimality Conditions in Multiobjective Programming," Systems Science and Mathematical Sciences, Vol. 1, 1988, pp. 184-190.

[22] F. J. Gould and J. W. Tolle, “A Necessary and Sufficient Qualification for Constrained Optimization,” SIAM Journal on Applied Mathematics, Vol. 20, No. 2, 1971, pp. 164-172. doi:10.1137/0120021

[23] G. Bigi and M. Pappalardo, "Regularity Conditions in Vector Optimization," Journal of Optimization Theory and Applications, Vol. 102, No. 1, 1999, pp. 83-96. doi:10.1023/A:1021890328184

[24] M. C. Maciel, S. A. Santos and G. N. Sottosanto, "Regularity Conditions in Differentiable Vector Optimization Revisited," Journal of Optimization Theory and Applications, Vol. 142, No. 2, 2009, pp. 385-398. doi:10.1007/s10957-009-9519-2

[25] K. H. Elster and J. Thierfelder, “Abstract Cone Approximations and Generalized Differentiability in Nonsmooth Optimization,” Optimization, Vol. 19, No. 3, 1988, pp. 315-341. doi:10.1080/02331938808843348

[26] G. Giorgi and A. Guerraggio, "On the Notion of Tangent Cone in Mathematical Programming," Optimization, Vol. 25, No. 1, 1992, pp. 11-23. doi:10.1080/02331939208843804

[27] J. Palata, "A Survey of Conical Approximations Used in Optimization,” Optimization, Vol. 20, No. 2, 1989, pp. 147-161. doi:10.1080/02331938908843424

[28] J. P. Penot, "A Characterization of Tangential Regularity,” Nonlinear Analysis, Methods \& Applications, Vol. 5, No. 6, 1981, pp. 625-643. doi:10.1016/0362-546X(81)90079-1

[29] Z. F. Li and S. Y. Wang, "Lagrange Multipliers and Saddle Points in Multiobjective Programming," Journal of Optimization Theory and Applications, Vol. 83, No. 1, 1994, pp. 63-81. doi:10.1007/BF02191762

[30] K. H. Elster and R. Nehse, "Optimality Conditions for Some Nonconvex Problems," Lecture Notes in Control and Information Sciences, Vol. 23, Springer Verlag, Berlin, 1980, pp. 1-9.

[31] M. Hayashi and K. Komiya, "Perfect Duality for Convexlike Programs," Journal of Optimization Theory and Applications, Vol. 38, No. 2, 1982, pp. 179-189. doi:10.1007/BF00934081

[32] V. Jeyakumar, "Convexlike Alternative Theorems and Mathematical Programming,” Journal of Optimization Theory and Applications, Vol. 16, No. 5, 1985, pp. 643652. doi:10.1080/02331938508843061 